\title{
LES MALADIES DES ÉCREVISSES, LEUR RECONNAISSANCE ET LA SURVEILLANCE SANITAIRE
DES POPULATIONS ASTACICOLES
}

A. VEY

Station de Recherches de Pathologie Comparee INRA-CNRS, 30380 ST. CHRISTOL

\section{RESUME}

Les écrevisses sont atteintes dans les populations naturelles comme en élevage par des maladies dues à des métazoaires parasites, des protozoaires, des bactéries et des champignons. Ces mortalités sont souvent liées à l'intervention des causes favorisantes ou à des infections multiples.

La reconnaissance des causes de lètalité chez les écrevisses est gérieralement complexe et nécessite l'intervention d'un Laboratoire. Les résultats de travaux de pathologie ont permis d'établir des fiches techniques et des protocoles de diagnostic facilitant ce travail d'identification. Cette situation est favorable à la mise en place rapide dun contrôle sanitaire des populations astacicoles.

Le développement des recherches sur les maladies des ecrevisses constituera un facteur essentiel pour la reussitc de la protection des popuiations naturelles, des operations de repeuplement et des tentatives d'aquaculture. II permettra aussi une meilleure compréhension des aspects pathologiques des equilibres en milieu aquatique.

\section{SUMMARY}

Diseases are caused in natural and artificial populations of crayfish by parasitoids. protozoan, bacteria and fungi. The death of the host is often related to the intervention of favoring factors or provoked by multiple infections.

The recognition of causes of death is often complicated and requires the participation of a laboratory. The results of experimental investigations have allowed to establish technical sheets and diagnosis protocols making this identification easier. This situation is favorable to a rapid application of a sanitary control of crayfish populations.

The development of researches on crayfish diseases vill be an essential factor fo the success of protection of natural populations. restoration operations and aquaculture It will also permit a better understanding of pathological aspects of equilibria in freshwaters 


\section{INTRODUCTION}

Les écrevisses, crustacés décapodes habitants des lacs et des rivières, ont retenu depuis longtemps l'interêt des pêcheurs, leurs qualitès gastronomiques en faisant des mets appréciès. Ce sont également des invertébrés importants du point de vue écologique car ils constituent des éléments des biocœnoses aquatiques exerçant leur action à différents niveaux de la chaine alimentaire

Malheureusement il a dejà èté constaté dans le passe, en particulier à la fin du $19^{\text {e }}$ siècle, des réductions de populations considérables s'étendant à la plus grande partie du territoire français. Dans une période plus récente, même si des épidèmies brutales et spectaculaires n'ont pas èté observées, les enquêtes effectuées périodiquement, en particulier la dernière dont les rèsultats ont èté publiès en 1980 (LAURENT et SUSCILLON, 1962 ; VIGNEUX, 1980), font ressortir une tendance à la raréfaction continue d'Atlantoastacus pallipes Ler. ou * écrevisse à pattes blanches*, alors qu'Astacus astacus L. n'est plus présent qu'au niveau de quelques biotopes restreints surtout au nord-est de notre pays. II est apparu que si des causes diverses interviennent dans cette régression des populations astacicoles, telles que les pollutions, les assecs en periode estivale, les modifications du milieu par pompage. curage ou rectification des cours d'eau. les pèches abusives et l'action des prédateurs, les maladies sont souvent mises en cause dans un tel processus (REBEYROL, 1978).

Devant cette situation la pêche des écrevisses a èté sèvèrement restreinte, et pour faire face aux besoins de la consommation it est fait appel aux importations qui portent sur environ 2000 tonnes par an, et concernent majoritairement Astacus leptodactylus Esch. provenant de Turquie (Anonyme, 1979).

Ces importations constituent une source d'approvisionnement onéreuse et incertaine, les pays exportateurs pouvant y mettre brutalement fin. La nécessité d'une production nationale couvrant notre demande intérieure s'est donc peu à peu imposee, et de nombreux scientifiques et amateurs ont commencè à s'intéresser à l'élevage des écrevisses au cours des dernières années. Or des problèmes pathologiques se sont déjà manifestés fréquemment, aussi bien dans les èlevages expérimentaux que dans les astacicultures ayant atteint un certain niveau de production, et ont été à l'origine de pertes considérables.

L'importance des processus pathologiques dans le domaine de l'astacologie a èté ressentie très tôt notamment par le Conseil Supérieur de la P'êche et par l'Institut National de la Recherche Agronomique. C'est ainsi que des observations sur les maladies des astacidae ont commencè à la Station de Pathologie Comparee INRA-CNRS à Saint-Chrictol à partir de 1966. L'intérêt porté à la pathologie des écreviśsss a conduit très rapidement à l'établissement d'une convention, qui a permis le développement actif des recherches dans le cadre diun programme defini en collaboration.

Dans le présent article seront exposés brièvement les résultats obtenus au cours de ces programmes concernant la mise en évidence et l'ètude des agents pathogènes attaquant les écrevisses et la caractérisation des maladies qu'ils provoquent, ainsi que leurs frolongements dans le domaine du diagnostic et du contrôle sanitaire. Des données de la littérature ainsi que des communications personnelles seront utilisées pour compléter certains points de cet exposé 


\section{ETAT DES CONNAISSANCES ACTUELLES SUR LES FRINCIPALES MALADIES}

\section{1) Mycoses}

Les infections dues à des champignons comptent parmi les maladies les plus frèquentes chez les écrevisses, et sont dues à des cryptogames très différents, aussi bien par leur position systematique que par leur pouvoir pathogène.

L'Aphanomycose ou "peste des écrevisses" est la maladie certainement la plus dangereuse pour les astacidae. Les premières èpidemies qui se sont manifestèes en Europe à partir de 1860 ont été attribuèes à une bactérie, Bacillus pestis astaci (HOFER, 1904). ces résultats étant invérifiables à l'heure actuelle. A partir de 1930 environ il est apparu que le responsable est en fait un champignon de l'ordre des Saprolegniales, Aphanomyces astaci Schikora (SCHAPERCLAUS, 1935; NYBELIN, 1936). Les attaques se produisent au niveau des parties minces et relativement tendres de la carapace, ciest-à-dire les membranes articulaires à la base des pattes, les membranes incorsegmentaires et le tégument de la face ventrale de l'abdomen, l'invasion des tissus internes étant très limitée.

Toutes les espèces européennes d'astacidae sont sensibles à $A$. astaci, alors que les espèces américaines sont hautement mais cependant non totalement résistantes. II faut noter à ce sujet que ces animaux * résistants - peuvent ètre atteints d'infections chroniques non mortelles et porteurs d'Aphanomyces (Fig. 1).

En France, la situation indiquee dans une précedente revue (VEY, 1979) s'est maintenue. La surveillance effectuée sur le terrain et les investigations en Laboratoire n'ont pas mis en évidence d'épizootie due à $A$. astaci dans les populations naturelles d'écrevisses autochtones. En ce qui concerne les animaux provenant d'èlevages ou d'installations de retrempage, seuls de rares échantillons de populations de Pacifastacus leniusculus Dana de provenance nord-américaine se sont révélès hautement suspects. Les dangers liès aux importations de sujets vivants sont donc particulièrement grands étant donné en particulier la possibilité de réintroduction et de dissémination de l'aphanomycose.

Plusieurs types d'infections fongiques sont causés par des champignons imparfaits du genre Fusarium. Récemment des mycoses atteignant les organes branchiaux et dues à ces agents cryptogamiques ont été decelées chez $A$. leptodactylus (VEY, 1978). Ces maladies s'accompagnent du développement d'importantes taches brun sombre sur les branchies. dues à une réaction de mélanisation dans les zones de pénétration du champignon. L'attaque reste localisée au niveau du tissu branchial (Fig. 2), les organes internes n'étant pas atteints, mais parvient cependant à provoquer la mort de l'animal. La penétration de ces Fusarium est favorisèe par l'existence de lésions sur les tuhules. 

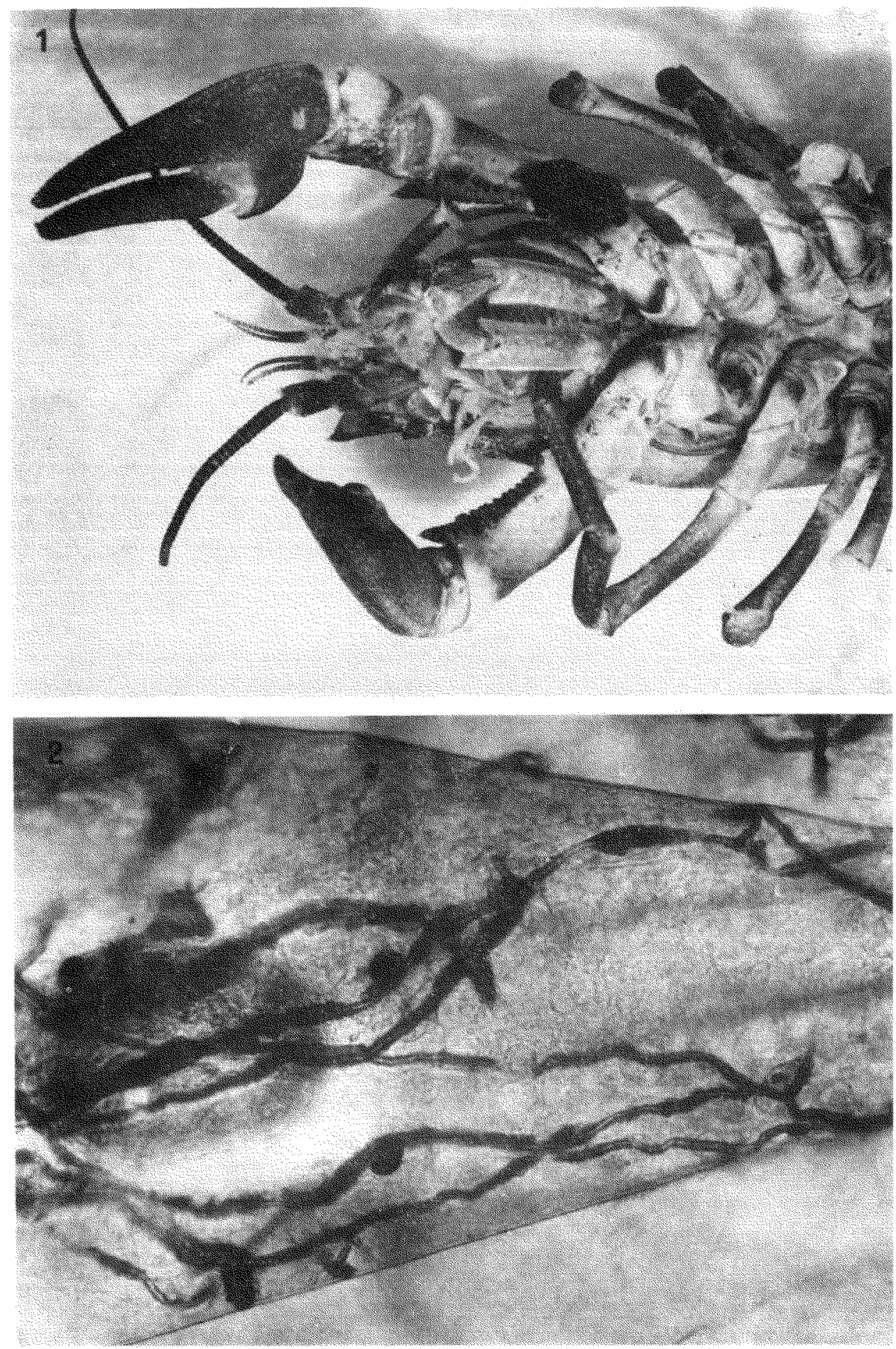

Fig. 1 - Infection à Aphanomyces astaci Schik. chez Pacifastacus leniusculus Dana. On remarque une tache noirâtre sur la pince gauche, correspondant à une zone d'attaque.

Fig. 2 - Filaments mélanisés de Fusarium sp. développés dans la branchie d'Astacus leptodactylus Esch. Montage tissu frais, non coloré. X 300. 
Les Fusarium sp. sont également aptes à attaquer les écrevisses en se développant dans la carapace et provoquant l'apparition de taches tégumentaires brunes qui peuvent couvrir la moitié de !'abjomen (Fig. 3). Cette invasion de la cuticule, qui touche aussi bien les zones membranaires que les parties calcifiées les plus epaisses, s'accompagne d'une croissance mycélienne interne se traduisant par l'apparition d'un amas volumineux de filaments et de cellules sanguines incluant certaines masses musculaires. La localisation des attaques, qui ne touchent que faiblement les organes vitaux, explique l'evolution lente de ce type de mala'die.

On peut rapprocher de ce type d'affection pathologique les a rouilles - décrites depuis longtemps, mais dont l'origine n'a pas encore été éclaircie. Les premières observations realisees nous ont permis de reconnaitre que les taches eccompagnant ce type de maladie contiennent parfois des èléments mycéliens alors que dautres ne semblent pas renfermer d'agent étranger.

Les saprolégniales sont des phycomycètes aquatiques souvent présents dans les eaux douces et qui n'attaquent qu'occasionellement les écrevisses. Le parasitisme par des Saprolegnia sp. se développant essentiellement au niveau tégumentaire a en effet été observé chez $A$. pallipes comme chez A. leptodactylus dans des conditions d'élevage ou de stockage (Fig. 4). Dans les différents cas étudiés l'analyse des conditions ayant régné au moment de la mort des animaux a permis de conclure effectivement à l'intervention de facteurs favorisants tels que faible oxygènation liée à un débit réduit, excès de farticules dans l'eau, ou aliinentation insuffisante prolongée. En outre, les essais d'infections expérimentales sur des animaux placés dans de bonnes conditions de milieu ont donné jusqu'à présent des résultats négatifs. ce qui confirme la pathogenécité réduite des souches de saprolégniales isolèes.

Ces champignons sont également à l'origine d'infections atteignant les pontes (VEY. 1977). Les œufs attaqués dégénèrent, prennent une coloration orangée et s'entourent d'une frange cotonneuse très développee. Les saprolégniacae s'installent probablement d'abord sur des œufs isolés en cours d'avortement ou lésés, puis parviennent à envahir une grande partie ou même la totalité de la ponte qui est recouverte par le mycelium et détruite. Les écrevisses elles-mêmes ne semblent pas être atteintes par les champignons jéveloppés sur leurs pontes.

\section{2) Bactérioses}

Des infections bactériennes se manifestent egalement dans la période actuelle et sont à l'origine de létalités chez les écrevisses (TOUMANOFF, 1965, 1936. 1967, 1939; VEY, BOEMARE, VAGO, 1975 : BOEMARE, VEY. 1977)

Un premier type d'affection à étiologie bactérienne se manifeste notamment chez A. pallipes en période estivale, ou chez $A$. astacus, sans apparition de symptôme caractéristique. II s'agit de bactérioses septicémiques typiques avec développement abondant des microorganismes dans le sang, en particulier au niveau du cœur et des branchies (Fig. 5). II se produit une infiltration des corps bactériens dans le muscle. les autres tissus n'étant fas atteints. Les agents responsables sont des bactèries bacilliformes gram négatif non $s ; 0-$ rulés, en particulier des Pseudomonas aeruginosa et $P$. putida

Un autre type de syndrome se caracterise par une multiplication intense de bactéries dans le tube digestif et surtout l'hépatopancréas (Fig. 6). Un passage de ces microorganismes à partir de ces organes dans la cavité generale ne se projuit quà un stade très tardif de la maladie. De telles infections s'accompagnent de lésions de l'hépatopancréas dans lequel des tubules dégénèrent, mèlanisent et s'entourent d'une importante reaction inflammatoire, ce qui conduit à la formation de kystes dans cet organe 

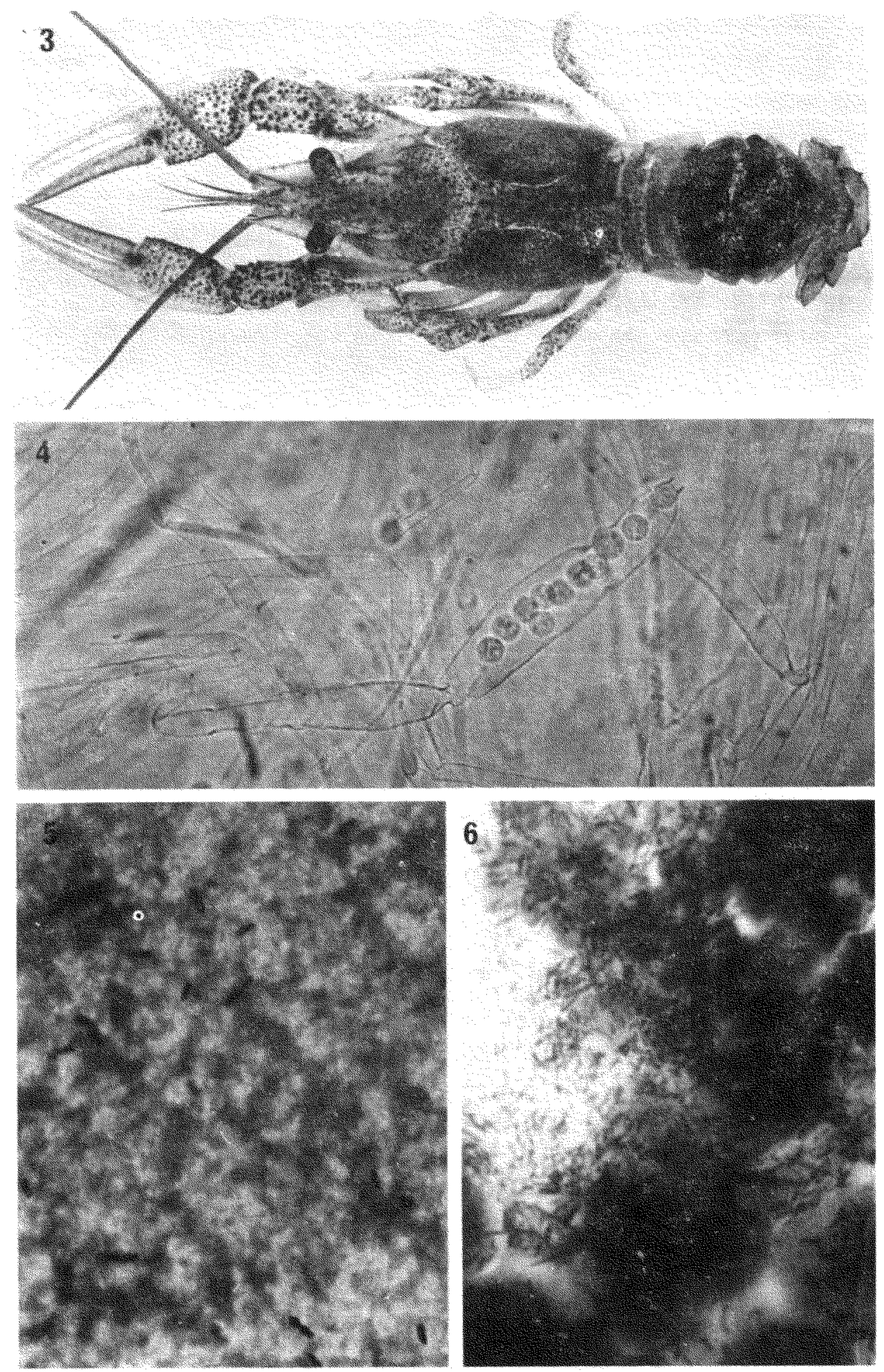

Fig. 3 - Large zone abdominale pigmentèe chez $A$. leptodactylus atteint d'infection tégumentaire à Fusarium sp.

Fig. 4 - Saprolegnia diclina Humphrey parasite d'Atlantoastacus pallipes Ler. Sporange provenant d'une culture sur graine de chanvre. $X 300$.

Fig. 5 - Bactéries dans l'hèmolymphe d'A. pallipes atteint de septicémie à $P$. fluorescens. Frottis, color May Grünwald-Giemsa. X 1100.

Fig. 6 - Tissu hépatopancréatique envahi par des bactéries dans un cas de maladie hépatointestinale. Frottis, color. May Grünwald-Giemsa. X 1100. 
Les agents responsables de telles maladies peuvent etre des Citrobacter sp. et des Pseudomonas sp.

\section{3) Parasitoses}

Des Protozoaires et Métazoaires divers ont èté considérés comme responsables de mortalités chez les écrevisses.

Parmi les métazoaires ectoparasites notes depuis longtemps on compte les Branchiobdella qui se présentent sous l'aspect de petites sangsues se fixant sur la cuticule d'écrevisses de différentes espèces par des ventouses. Ce:s vers peuvent pulluler et atteindre un effectif d'une centaine par animal parasité. Ils ne sont généralement pas considérés comme des causes de mortalité, et les cas où ils ont été mis en cause restent à confirmer. Dans les cas que nous avons examinès il n'a pas èté constatè d'affaiblissement excessif des écrevisses atteintes, susceptible à lui seul de provoquer leur mort, et la prédation sur les œufs a paru porter seulement sur les œuís dégénérés. Il faut cependant retenir la possibilité d'un effet nocif en cas de pullulation intense des Branchiobdella, surtout si ces parasites se fixent essentiellement au niveau des branchies.

En ce qui concerne les protozoonoses, une maladie importante des écrevisses est causée par la microsporidie Thelohania contejeani Henneguy (HENNEGUY et THELOHAN, 1892), qui se développe chez $A$. astacus et $A$. pallipes. Uno coloration blanc laiteux due $\exists$ la transformation du tissu musculaire apparait sur la face ventrale de l'animal farasité. Les examens microscopiques de frottis et de coupes histologiques, ainsi que les études en microscopie électronique, ont permis de reconnaitre et de décrire en détail les différents stades de développement dans l'hôte. et de préciser la localisation des attaques tissulaires (MAURAND et VEY 1973). L'action letale de Thelohania est surtout liée à la destruction des fibrilles musculaires, tous les muscles y compris ceux de la paroi cardiaque étant finalement attaqués, mais découle probab!ement aussi du développement de ce parasite dans la masse cerébrale (Fig. 7)

$T$. conteneani, répandue en Europe, est également commune en France. Nous l'avons rencontrée fréquemment dans les populations d'A. pallipes du Massif Central.

Si l'impact de Thelohania dans la nature est certain, ceci parait dû moins au fait que ce parasite provoque des épizooties avec mortalités massives qu'à son action de type enzootique, le taux d'animaux atteints variant souvent entre 1 et $20 \%$. Cependant certaines données anciennes indiquant des niveaux de parasitisme plus elevés, en particulier tans les lacs (DOLLFUS, 1935; SCHAPERCLAUS, 1957), ont éte confirmées par des observations récentes suggèrant même de véritables mortalités épizootiques dues à Thelohania dans de petits ruisseaux particuliers (CHAISEMARTIN, comm. pers.). II faut également insister sur le danger que représente $T$. contejeani pour les expériences d'élevage des écrevisses européennes (VEY et VAGO, 1972; VEY, 1976).

Une autre affection est due à un parasite decouvert lui aussi très anciennement (HAECKEL, 1857) et dénommé Psorospermium haeckeli par HILGENDORF (1883). P. haeckeli est présent dans de nombreux pays europeens. Particulièrement fréquent chez $A$. leptodactylus importé de Turquie, cet endoparasite attaque aussi d'autres espèces euróéennes ou nord-américaines : A. astacus, Orconectes limosus Raff. et $P$. leniusculus, et atteint aussi bien les adultes que les juvéniles.

$P$. haeckeli se prèsente dans le tissu conjonctif des animaux atteints sous forme d'éléments en majoritè d'aspect homogène, ovoides de $35 \times 70 \mu$ en moyenne. La paroi de ce stade du parasite est très épaisse et de structure caractéristique. Elle compte en effet 4 couches, la seconde montrant une disposition en plaques aux bords amincis, séparees par des sillons (Fig. 8 et 9). Les autres etapes du développement chcz l'écrevisse. ou chez un autre hôte, ne sont pas connues avec certitude.

La pathogénicité de Psorospernium a été longtemps discutée, plusieurs auteurs considérant que cet agent ne causait pas de dommage notable chez son hôte. En fait nos observations (VEY, 1978 b) ont permis de constater que tous les animaux atteints meurent finalement après un délai qui peut atteindre plusieurs mois. Or remarque également que les mortalités des animaux porteurs de $P$. haeckeli sont souvent synchronisées avec les mues. 


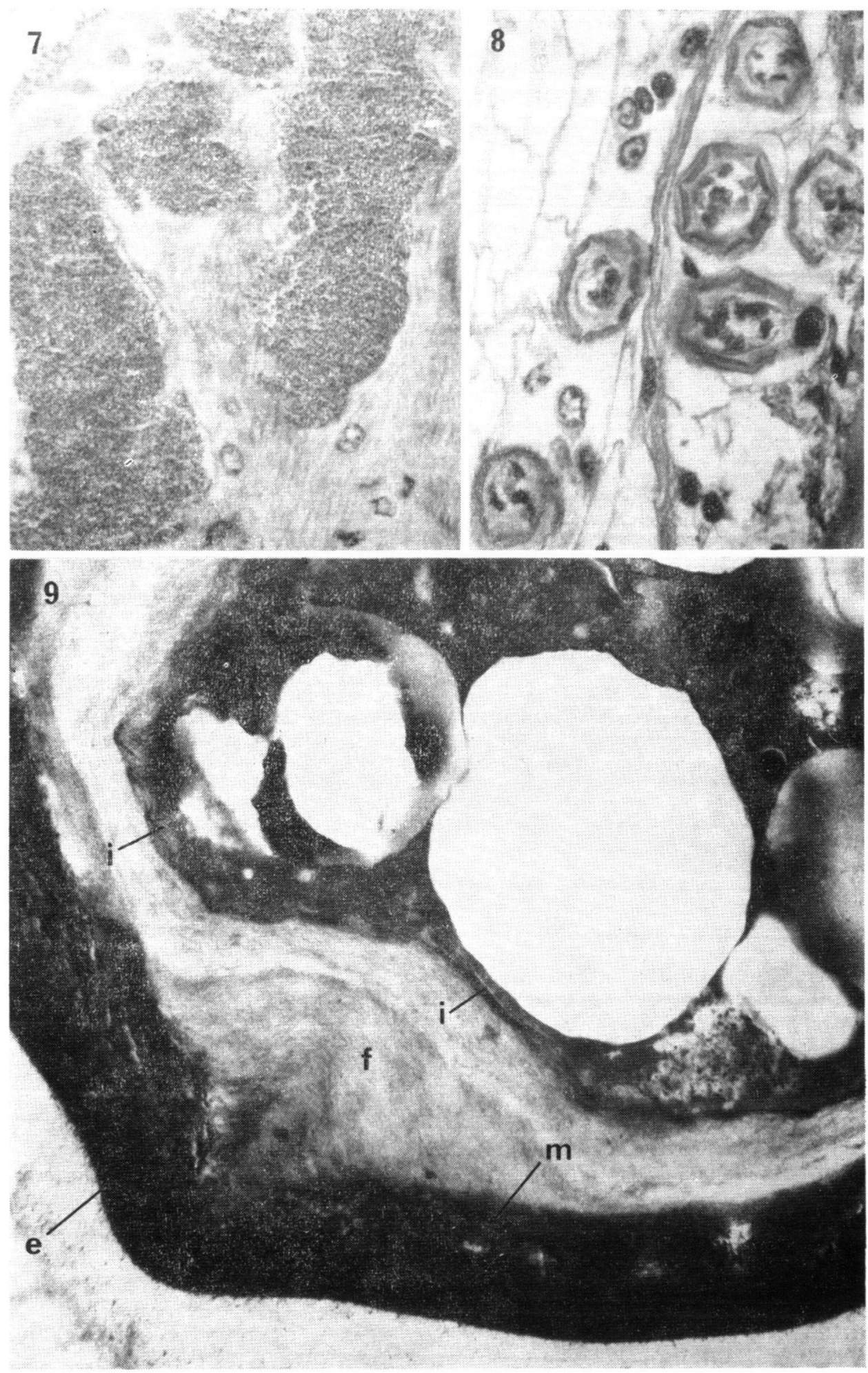

Fig. 7 - Developpement de Thelohania contejeani Henneguy dans le muscle d'A. pallipes Coupe histol., color. Armargier-Vago. X 260.

Fig. 8 - Eléments de Psorospermium haeckeli Hilg. dans le tissu conjonctif sous cu'iculaire d'A. leptodactylus. Coupe Histol., color. Hémalum-picro-indigocarmin. X 300 .

Fig. 9 - Vue détaillée de la structure de $P$. haeckeli. Dans la paroi on reconnait la couche externe dense (e), la couche moyenne en plaques ( $i:)$, la région fibrillaire ( $f$ ) et la membrane interne (i). Micr. electron. X 9000. 


\section{4) Infections virales}

Bien que des contrôles virologiques aient été frequemment effectués dans le cajre d'examens pathologiques d'écrevisses malades ou suspectes, il n'a pas été découvert de maladie d'origine virale ou rickettsienne chez les astacidae. II faut cependant noter que dans le domaine de la pathologie marine où des recherches systématiques ont été effectuees, de nombreux agents viraux appartenant à différents groupes taxonomiques ont èté découverts chez les décapodes (BONAMI. 1976). II faut en outre considerer que pour un certain nombre de mortalités les résultats négatifs des examens parasitologiques, mycologiques et bactériologiques et l'absence de toute indication de pollution chimique conduisnni à envisager une étiologie virale. La poursuite des recherches approfondies sur les maladies d'écrevisses conduira donc probablement à la decouverte te létalités associées à des agents de type viral.

\section{5) Infections multiples, facteurs favorisants :}

Comme cela se produit chez les autres animaux, les mortalités chez les écrevisses ne sont pas toujours d'un type simple, c'est-à-dire dues à une cause unique. En effet différents agents facultativement pathogènes, notamment des bactéries ou des champignons, $n$ 'interviennent que dans la mesure où s'exercent dans un premier lemps des causes íavorisantes. et entrent en jeu de manière secondaire dans des complexes pathologiques. L'affaiblissement constituant le facteur favorisant peut être lui-mème de nature parasitologique ou microbien On assiste en effet à des infections doubles thelohaniose-saprolégniose, ou psorospermiosemycoses de la carapace. Les maladies complexes observées chez l'écrevisse peuvent d'ailleurs faire intervenir un plus grand nombre d'agents. Ainsi des cas d'infections multiples qui peuvent faire intervenir Psorospermium, des bactéries septicémiques, et des Fusariums sp. ou des Saprolegnia sp. ont été observès récemment chez A. leptodactylus. L'enchainement des differentes phases de ce complexe et le rôle joue par chacun des agerits font actuellement l'objet de recherches expérimentales.

La première phase des enchainements constatés chez les écrevisses est souvent constituee par une blessure tégumentaire. Les différentes infections a Fusarium sp. sonit favorisees par des lésions de la cuticule, alors que les bactéries responsables de septicémies pénètrent au niveau de ces traumatismes (VEY, BOEMARE, VAGO, 197j: VEY, 1978 a). De même les saprolegniales semblent bien s'installer d'abord sur des œ.jís lésés ou en cours davortement.

Les qualités du milieu aquatique jouent également un rôlc, même pour des agent: relativement indépendants de tels facteurs, comme Aphanomyces et Thelohonia. On notera. pour l'aphanomycose, l'importance de la richesse en sels minéraux, spécialcment en calcium, et la possibilité d'une action défavorable des pollutions organiques sur l'etabliss:c ment de l'infection (UNESTAM, 1969 a et b).

Cet effet des facteurs du milieu peut être plus no:ablu encorc, far exeriole pour los déperissements fongiques des pontes. Une abondance excessive de particules déposées sur le fond ou en suspension dans l'eau influence le dévoloppenont de ces affection: qui sont alors fréquentes. La ponte de certaines des femelles exposées à ces conditions ert d'ailieur. perturbée, et la présence d'œufs avortant après leur depôt (VEY, 1977) favorió certainement l'établissement de ces mycoses. En outre en terrain argileux, une tells abondanco de particules s'accompagne de la formation autour des branchies dune veritable gangue terrcuse qui entraine des perturbations res,3iratoires et permet la manifestation d'attaques par les $\quad: a$ prolégniales au niveau branchial

En ce qui concerne les bactérioses. les condition; estivales sont très propiccs cai elies favorisent un développement rapide du germe du fait des temperatures élevées, et tendent à affaiblir l'animal qui souffre en particulier de mauvaises conditions d'oxygénation (VEY, BOEMARE: VAGO, 1975) 


\section{RECONNAISSANCE DES CAUSES DE MORTALITE}

Un observateur, tel qu'un garde-pêche ou un astaciculteur, ne pourra identifier quiun nombre très réduit de maladies grâce à un examen externe à l'œil nu ou avec la loupe. complété par une observation des branchies après découpage au ciseau du volet latéral de carapace cephalothoracique. Ceci est possible lorsque l'agent entrainant la mortalité est visible sur l'animal. II en est ainsi pour le parasitisme par les Branchiobdella sp. qui apparaissent comme de petits vers rosâtres de quelques millimètres de long. Le parasitisme par les saprolégniales peut être également aisément décelé, en particulier au niveau des pontes car les filaments mycéliens se développent abondamment à la surface des œufs qu'ils entourent d'une épaisse couronne blanchâtre donnant l'aspect de mousse. Lorsque l'infection touche les écrevisses elles-mêmes une croissance fongique externe est également visible. bien que plus discrète. Lorsque l'on remarque de telles attaques fongiques, il est utile de s'assurer plus précisément de l'identite du champignon, et suitout de vérifier si ce phycomycète est bien l'agent étiologique de la mortalité, ou s'il n'intervient que secondairement à la suite de causes primarres favorisantes.

Dans tous les autres cas un examen simple ne permetira que de soujçonner l'intervention d'un parasite ou d'un germe pathogène determiné, ou méme ne fournira pas d'orientation valable. L'intervention d'un Laboratoire sera alors néces'saire.

Parmi les affections s'accompagnant de signes caracteristiques permettant de parvenir à un fort degré de présomption figure la thélohaniose. La coloration blanc-laiteuse du muscle visible au travers de la cuticule abdominale ventrale est assez typique pour être à l'origine: d'appellations courantes comme "maladie de la porcelaine. Neanmoins il se produit aussi sur tous les cadavres un char.gement de teinte du tissu musculaire, liè à la décomposition, et qui, bien que moins marque. peut conduire à une confusion. En outre l'apparition de colorations très comparables a été notèe dans d'autres affections, dues notamment à des champignons levuriformes. If sera donc nécessaire d'effectuer des examens microscopiques de montages et frottis de tissu musculaire afin de vérifier que celui-ci renferme bien en abondance des éléments ovoides de 3,5-4 $\times 2 \mu$, contenant un filament dévaginable. que l'on pourra par conséquent identifier comme des spores de microsporidie. Enfin, un examen plus complet permettra d'établir si les spores se forment par groupes de 8 à l'intérieur d'une même enveloppe, comme chez Thelohania, ou isolément comme dans le genre Nosema.

Plusieurs affections à étiologie fongique s'accompagnent aussi de signes externes susceptibles de favoriser leur détection. La présence de larges taches brun sombre sur les branchies. de même que le développement d'importantes zones tegumentaires également brunes, sont souvent dûs à des Fusarium sp. La présomption de mycose branchiale ou cuticulaire est à vérifier tout d'abord en recherchant la présence d'éléments mycéliens dans des fragments de tissus paraissant atteints, par des examens microscopiques. Ensuite, si des agents fongiques sont effectivement décelés leur identification nécessitera presque toujours leur isolement par mise en culture sur milieux mycologiques en vue d'examens morphologiques. Cependant, dans les infections au niveau des branchies une identification rapide du cryptogame au niveau du genre, ne nécessitant pas de faire appel aux méthodes micro-biologiques. peut être parfois réalisée, dans la mesure où le Fusarium produit des conidies caractéristiques dans et à la surface des tubules au cours des stades ultimes de la maladie.

Le diagnostic de l'aphanomycose présente différents points de difficulté et s'avère l'un des plus complexes. L'examen externe donne d'assez bonnes indications chez les espèces dites résistantes puisqu'au niveau d'articulations et de la cuticule abdominale ventrale se développent des taches brun très sombre localisées à ces parties minces du tégument. Par contre, chez les espèces hautement sensibles les signes d'attaque au niveau des mêmes localisations sont plus discrètes, alors qu'en fin de maladie et sur le cadavre un feutrage blanchâtre court se développe au niveau des régions de l'exosequelette attaquées ainsi que su: les yeux. Il sera donc nécessaire de s'assurer de la présence, dans les parties de cuticule suspectes, de filaments larges, de 5 à $10 \mu$ de diamètre, à bouts arrondis, avec des ramifications approximativement perpendiculaires. L'identification de l'agent cryptogamique ainsi repéré au genre Aphanomyces pourra être réalisèe grâce à son isolement sur milieu artificiel adapté et à la préparation de cultures particulières permettant d'obtenir d'abondants zoosporanges et zoosfores (UNESTAM, 1969 a). L'existence de sporanges cy'indriques, non renflés, conte- 
nant une seule rangée de zoospores sortant par une ouverture terminale, permet de rattacher le champignon obtenu en culture au genre Aphanomyces. II est difficile de s'assurer qu'un isolat d'Aphanomyces appartient bien à l'espèce $A$. astaci, car les différentes espèces de ce genre sont morphologiquement très proches et difficiles à determiner. Par contre, $A$. astaci étant le seul à manifester une forte pathogénicité vis-à-vis des écrevisses on devra procéder à un test biologique mettant en contact des sujets atteints ou le champignon isole avec des A. pallipes ou des $A$. astacus hautement sensibles. Ce dernier contrôle est particulièrement important pour des animaux qui, comme Pacifastacus leniusculus Dana, peuvent ètre porteurs sans que leur vitalité soit nettement affectee. d'autant plus que les essais d'isolement d'Aphanomyces à partir de taches de leur tégument connaissent souvent un échec (SODER. HALL, comm. pers.).

L'existence de signes externes et internes peut également orienter le diagnostic dans certains cas de bactérioses. En effet, l'apparition d'une coloration brun clair de l'hémolymphe, visible sur la face ventrale de l'abdomen, une coloration et une consistance anormales de l'hépatopancréas, et la présence éventuelle de nodules mélanisés dans cet organe accompagnent les infections de type entérite caractérisées par un abondant développement de microorganismes dans le tube digestif et ses annexes La confirmation de l'intervention de bacteries dans ce type de mortalité est obtenue aisément par l'examen de frottis d'hépatopancréas.

Enfin, dans certains types de mortalitès qui ne sont pas accompagnés de symptômes ou lésions on est conduit à rechercher, par exemple, le développement de bactéries septicèmiques dans le sang, en particulier par la mise en culture de prélèvements aseptiques d'hémolymphe sur milieux bactériologiques. Dans de tels cas il doit être également procédé à la recherche de Psorospermium dans des explants de tissu conjonctıf prélevé au niveau des volets latéraux de la carapace, ou de la zone médio-dorsale du céphalothorax. Cependant la présence de $P$. haeckeli s'accompagne parfois de taches orangées de la carapace, dont la présence sera une incitation à rechercher particulièrement ce parasite.

Tenu compte du fait qu'un diagnostic ne pourra être portè que par un Laboratoire dans la plupart des cas de maladie, un tel établissement doit être contacté rapidement par toute personne ayant connaissance d'anomalies ou de mortalités. L'observateur doit également établir une fiche de renseignements qui constitueront des éléments importants pour l'établissement et l'interprétation du diagnostic. Ces donnees concerneront particulièrement le lieu et la date à laquelle s'est manifestée la maladie, les anomalies du comportement ou de l'aspect des animaux notées au cours de l'examen des écrevisses atteintes, les valeurs des paramètres du milieu aquatique et l'existence de pollutions organiques ou chimiques. La réussite des études pathologiques est avant tout conditionnée par l'expédition, effectuée dans de bonnes conditions, d'animaux typiquement atteints mais pas encore morts, accompagnés si possible d'individus paraissant sains, et éventuellement de cadavres. Lorsque les animaux sont encore vigoureux, on peut les placer dans un emballage résistant tel quine caissette en bois contenant des plantes ou un substrat naturel humidifie. $\mathrm{Si}$ les sujets malades sont très affaiblis, il peut être préférable de prolonger au maximum leur longévité en employant un emballage isolant contenant un sac à glace bien maintenu en place.

On peut compléter l'expédition d'animaux, ou tenter dans une certaine mesure de la remplacer, s'il n'y a pas de sujet vivant disponible, par la confection de frottis de tissus ou la fixation soit de parties du corps soit d'organes precis dans des liquides fixateurs en vue d'examens histologiques.

En outre, après une épidémie il est important d'observer si la maladie persiste, pendant quelle durée, et si elle réapparait au cours des mois et des années suivantes, car une telle surveillance est susceptible de nous informer sur la conservation du germe dans le milieu, sur ses réintroductions, et sur la nature des causes favorisant le déclenchement des épizooties.

\section{CONTROLE SANITAIRE}

Au cours des dernières annèes nous avons constaté un accroissement des examens pathologiques à effectuer sur des écrevisses, résultant d'un intérêt plus marqué pour ces animaux, en particulier du souci de proteger les populations naiurelles encore existantes et de 
réaliser des opérations de restauration des peuplements astacicoles efficaces. Cette tendance nous parait d'ailleurs susceptible de se renforcer dans l'aven:r.

De même, étant donné la manifestation de fréquentes mortalités dans les établissements d'astaciculture expérimentale ou de production, de nombreux cas ou les maladies semblaient pouvoir être mises en cause nous ont été soumis.

On notera aussi que les multiples importations d'écrevisses qui sont effectuées en France, que ce soit en vue de la commercialisation pour la consommation, de l'èlevage, ou du repeuplement, constituent un risque élevè sur le plan sanitaire, les astacidae n'ètant contrôlés actuellement que du point de vue de l'absence de dainger en tant qu'aliment

La situation que nous venons de decrire rend nécessaira la mise sur pied à bref délai d'un contrôle sanitaire systematique. Or, depuis peu, on dispose de bonnes bases pour la réalisation d'un tel contrôle, car les travaux déjà effectués dans notre Station dans le domaine de la pathologie astacicole ont permis d'accroitre notablement les connaissances, qu'il s'agisse d'affections déjà découvertes antérieurement ou nouvelles. Ces recherches ont atteint un staje de maturite qui a permis de rassembler et présenter un certain nombre de données sous forme de fiches techniques ou de protocoles de reconnaissance consacrés aux maladies les plus importantes et les mieux connues. Ces documents, fruits de la cooperation de la Station de Pathologie Comparée INRA et du CSP. sont directement utilisables dans la pratique. Ils constituent en particulier des éléments d'information qui ont été mis à la disposition de la Direction des Services Véterinaires qui met actuellement au point un protocole de contrôle sanitaire applicable aux astacicultures comme aux installations de retrempage, et tenant compe de l'avancement des connaissances.

L'organisation en cours de mise en place prévoit l'intervention sur le terrain des Directions de Services Vetérinaires départementales, et la participation sur le plan des analyses vétérinaires du Laboratoire Central de Maisons-Alfort. Cette action est menée en collaboration etroite avec la Station de Recherches de Pathologie Comparée INRA.CNRS de SaintChristol qui développe en même temps des programmes de recherche détaillés concernant les maladies des astacidae, et des sujets d'investigation nouveaux à partir de cas de mortalité faisant intervenir des germes mal connus, ou que le diagnostic courant n'aura pas permis d'élucider de manière satisfaisante.

\section{CONCLUSION}

Les recherches conduites sur les maladies des écrevisses montrent que ces invertébrés sont atteints dans la nature aussi bien que dans les conáitions d'élevage par des agents divers : métazoaires parasites, protozoaires, champignons, bactéries, alors qu'il n'a pas encore éte mis en évidence de manière certaine d'affection d'origine virale.

Souvent les mortalites survenant chez les astacidae presentent un caractère complexe. Ainsi un certain nombre de microorganismes de nature fongique ou bactérienne ne parviennent à provoquer des infections mortelles que si une action favorisante de facteurs concernant les qualités du milieu, l'alimentation, ou liés à la densité de population, affaiblit les animaux et diminue leur résistance. En outre des infections multiples faisant intervenir plusieurs agents se manifestent egalement. Les modalités d'intervention simultanée ou en enchainement des differents germes participant à ces complexes sont actuellement en cours d'étude.

Les résultats des investigations que nous avons effectuees ont des conséquences directement utilisables dans la pratique. En effet, ces travaux ont en particulier permis de mettre au point des documents techniques concernant les maladies des astacidae et leur reconnaissance. Ces textes seront complétés par une publication plus importante en volumes en cours de rédaction, dont la nécessité est apparue clairement. Une telle brochure traitera en détail de l'ensemble des problèmes pathologiques connus chez les écrevisses et sera abondamment illustree afin de constituer à la fois un moyen d'information et un outil pédagogique s'adressant à un large public. 
La vulgarisation des informations n'est cependant pas susceptible de permettre lidentification exacte et complète de la majorité des affections pathologiques touchant les astacidae par tout observateur non spécialisé.

L'intervention du Laboratoire spécialisé restera dono généralement nécessaire, notamment en l'absence de signe apparent caractéristique.

Les progrès realisés dans la connaissance des maladies des écrevisses, principalement grâce aux recherches développees à la Station de Recherches de Pathologie Comparée de Saint-Christol sous l'égide de I'INRA et du CSP créent une situation favorable pour la mise en place d'un contrôle sanitaire efficace des établisscments astacicoles. Cette surveillance serait effectuee par les Services Vétérinaires, en collaboration avec le Laboratoire de la Station de Pathologie Comparée INRA-CNRS specialise en pathologie astacicole.

La poursuite active des recherches concernant les mantestations pathologiques chez les astacidae, ainsi que la mise en œuvre d'une surveillance sanitaire syztematique, constitueront des facteurs essentiels à la reussite des operations de protection des populations naturelles et de repeuplement aussi bien que des tentatives d'aquaculture appliquées aux écrevisses.

Dans le domaine de la protection de l'environnement le problème d'équilibre Jans les lacs et dans les eaux courantes est de plus en plus mis en avant. Dans ce contexte les peuplements d'écrevisses sont très importants, même pour des aspects pratiques comme la pêche. Dans l'avenir l'étude des cas pathologiques sera de ce fait encore plus nécessaire que par le passé. Le Laboratoire de Saint-Christol est bien engagé, en collaboration avec le CSP et le Ministère de l'Environnement. dans les études des aspects pathologiques de ces équilibres, notamment dans celles des conséquences pathologiques des interventions humaines dans le milieu aquatique.

\section{REFERENCES}

ANONYME, 1979. Importations et exportations d'écrevisses pour les trois dernières années. La Pisciculture Française, 56, p. 61.

BOEMARE N. et VEY A., 1977. Etude des souches bactériennes isolées d'écrevisses Atlantoastacus pallipes Lereboullet atteintes de septicemies et d'affections hépato-intesinales. Ann. Hydrobiol... 8, 153-162.

BONAMI J.R., 1976. Les maladies virales des crustacés et des mollusques. Oceanis, 3 , 154-175.

DOLLFUS R., 1935. Thelohaniose de l'écrevisse (Astacus pallipes Lereb.) à Richelieu (Indre et Loire). Bull. Soc. centr. Aquic. et Pêche. 42, 119.

HAECKEL E. 1857. Uber die Gewebe des Flusskresbses. Arch. f. Anat. Physiol., 24, 561-562.

HENNEGUY G. et THELOHAN D., 1892. Myxosporidies parasites des muscles chez quelques crustacés décapodes. Ann. Micrographie, 4, 617-641.

HILGEUDORF F. 1883. Bemerkungen über die sogennante krebspest, inbesondere über Psorospermium haeckeli. Sitzungsber. Ges. naturf., 9. 179-183.

HOFER B., 1904 Handbuch der Fischkrankheiten. Verlag der Allg. Fischerei-Zeitung, München.

LAURENT P.J. et SUSCILLON M., 1962. Les ecrevisses en France. Amnales Station Centrale Hydrobiol. appl., 9, 335-395.

MAURAND J. et VEY A., 1973. Etude histopathologique et ultrastructurale de Thelohania contejeani Henneguy (Microsporida, Nosematidae) parasite de l'ecrevisse Austropotamobius pallipes Lerebou!let. Ann. Parasitol. Hum, et Cumo., 48. 411-421.

NYBELIN O. 1936. Untersuchungen über die Ursache der in Schweden gegenvartig vorkommenden Krebspest. Rep. Inst. Freshw. Res. Drottningholm, 9, 3-29. 
REBEYROL Y., 1978. Les écrevisses malades de la peste. Le Monde $N^{\circ} 10535,13$

SCHAPERCLAUS W., 1935. Die Ursache der pertartigen Krebssterben. Zeitsch. Fischerei. 33. 343-366.

SCHAPERCLAUS W., 1957. Fischkrankheiten, Akademie verlag. Berlin, 426-435.

TOUMANOFF C., 1965. Infections bactériennes chez les écrevisses. Premiere note: Protéoses. Bull. Fr. Piscic., 219. 41-65.

TOUMANOFF C., 1966. Infections bactériennes chez les écrevisses. Deuxième note : Citrobacter, Enterobacter. Bull. Fr. Piscic., 221, 117-133.

TOUMANOFF C., 1967. Infections bactériennes chez les écrevisses. Troisieme note : Pseudomonadacae : Pseudomonas alcaligenes Monias 1928 et Alcaligenes (Bacillus faecalis alcaligenes Petruschky 1896) et quelques bactéries proches incertae sedis. Bull. Fr Piscic. 227, 45-55

TOUMANOFF, C. 1968. Infections bactériennes chez les écrevisses. Troisième note : Pseudomonadacae : Pseudomonas, Alcaligenes et quelques bacteries proches (suite et fin). Bull. fr. Piscic., 228, 102-111.

UNESTAM T., 1969 a. On the adaptation of Aphanomyces astaci as a parasite. Physiol. Plantarum, 22, 221-235.

UNESTAM T., $1969 \mathrm{~b}$. On the physiology of zoospore production in Aphanomyces astaci. Physiol. Plantarum, 22, 236-245.

VEY A., 1976. Pathologie des écrevisses. La Pisciculture Flançaise, 48, 56-59

VEY A., 1977. Studies on the pathology of crayfish under rearing conditions. Freshwater Crayfish, 3, 311-319. O. Lindqvist Edit. Université de Kuopio, Finlande

VEY A., 1978 a. Infections fongiques chez l'écrevisse Pontastacus leptodactylus. Freshwater Crayfish, 4, 403-410. P.s. LAURENT Edit Institut National de la Recherche Agronomique. Thonon les Bains.

VEY A., 1978 b. Recherches sur une maladie des écrevisses due au parasite Psorospermium haeckeli Hilgendorf. Freshwater Crayfish, 4, 411-418, P.J. LAURENT Edit. Institut National de la Recherche Agronomique. Thonon les Ba!ns.

VEY A., 1979. Aspects fondamentaux et pratiques des recherches actuelles sur les maladies des écrevisses. La Pisciculture Française, 56, 41-45.

VEY A. et VAGO C., 1972. Recherches sur les maladies des écrevisses en France. Ann Hydrobiol. INRA, 3, 59-64.

VEY A. BOEMARE N. et VAGO C., 1975. Recherches sur les mala'dies bacteriennes de l'écrevisse Atlantoastacus pallipes Lereboullet. Freshwater Crayfish, 2, J.W. AVAULT Edit, Louisiana State University, Bāton-Rouge.

VIGNEUX D., 1980. Enquête sur les écrevisses en France, Conseil Supérieur de la Pêche, Paris. 